\title{
Structural insights into the enzymatic mechanism of lytic polysaccharide monooxygenases
}

\section{Flora Meilleur}

\author{
NC State / ORNL, Raleigh, United States of America \\ fmeille@ncsu.edu
}

Lytic polysaccharide monooxygenases (LPMOs) have been intensely studied since their first characterization in 2010 as a unique class of copper enzymes capable of oxidizing carbohydrates. LPMOs require the input of electrons and of $\mathrm{O}_{2}$ or $\mathrm{H}_{2} \mathrm{O}_{2}$ to achieve hydroxylation of one carbon in the glycosidic bond. We focus on three aspects of the LPMO's reaction mechanism: 1) What are the structural determinants of $\mathrm{O}_{2}$ and $\mathrm{H}_{2} \mathrm{O}_{2}$ binding? 2) How do conserved second shell residues contribute to activity? 3) Does the $\mathrm{O}_{2}$ based mechanism follow a superoxyl, hydroperoxyl or oxyl catalytic pathway? The ability to pinpoint hydrogen atoms to determine protonation states at and around the active site through the catalytic pathway is key to decipher the chemistry catalyzed by LPMOs. To achieve this, we combine high resolution X-ray and neutron protein crystallography to deliver precise, all atom structures of key reaction intermediates that can reveal i) the positions and interactions of all hydrogen atoms in the enzyme, ii) atomistic details of the active site without perturbing the metal oxidation state, and iii) the chemical nature of the activated dioxygen species coordinated to the active site copper. We will present our recent X-ray and neutron crystallographic studies that provide new insights into the LPMO mechanism.

Keywords: monooxygenase, $\mathrm{O} 2$ activation, protonation state, neutron crystallography 